\title{
An approach to the transected umbilical vein catheter: a case series
}

\author{
A Bairagi, AG Maharaj \\ Department of Paediatric Surgery, Inkosi Albert Luthuli Central Hospital, Durban, South Africa \\ Corresponding author: Anjana Bairagi (anjana.bairagi@gmail.com)
}

\begin{abstract}
SUMMARY
Transected umbilical vein catheters (UVC) are potentially fatal and associated with considerable morbidity. Timely identification and catheter retrieval are crucial. The successful surgical managment of three neonates referred to Inkosi Albert Luthuli Central Hospital with retained UVCs and a proposed algorithmic approach to manage these patients is described.
\end{abstract}

KEYWORDS: umbilical vein catheter, transected, fracture, surgical retrieval

S Afr J Surg 2019;57(1) http://dx.doi.org/10.17159/2078-5151/2018/v57n1a2631

\section{Case One}

A 7-day, $1.7 \mathrm{~kg}$ premature female delivered at 32 weeks was referred with a retained umbilical vein catheter (UVC). The UVC was inserted for intravenous fluid requirements and was accidentally transected on removal. An unsuccessful local exploration and retrieval attempt was reported by the referring doctor. The infant showed no signs of respiratory distress, was haemodynamically stable with a blood pressure $(83 / 50 \mathrm{mmHg})$ and heart rate $(131 \mathrm{bpm})$. Examination revealed a row of nylon sutures, dried blood at the umbilicus and no visible catheter. Preoperative investigations showed a normal full blood count (FBC), Urea and Electrolytes (UE) and International Normalised Ratio (INR). An abdominal radiograph demonstrated the UVC tip position to be at the origin of the right internal jugular vein. A mini-laparotomy incision was used to isolate and clamp the umbilical vein distal to UVC. After venotomy, the UVC was easily identified, grasped and retrieved. The patient had an unremarkable postoperative course and was discharged to base hospital the following the day.

\section{Case Two}

A term, female neonate was referred with a retained UVC six days after birth. The cannula had fractured whilst being removed prior to discharge from the referral hospital. The admission UE and FBC were normal. The Liver Function Test (LFT) showed total serum bilirubin (TSB) of $121.0 \mu \mathrm{mol} / \mathrm{L}$ and GGT of $375 \mathrm{U} / \mathrm{L}$. The UVC was not visible on biplanar abdominal radiographs and under intraoperative fluoroscopic guidance, identification was difficult. The umbilical vein was located via a U-shaped periumbilical incision, then clamped and divided. An intact UVC was retrieved and umbilical vein transfixed with a 4-0 VICRYL $\AA$ (polyglactin 910, ETHICON, USA) suture. Postoperative recovery was unremarkable and the infant was discharged to the base hospital later the same day.

\section{Case Three}

A $1.2 \mathrm{~kg}$ male infant delivered at 30 weeks gestation, who required ventilation for 48 hours and phototherapy for neonatal jaundice, presented at 9 weeks of age for elective bilateral inguinal herniotomies. An ejection systolic murmur was noted at the preoperative assessment.

The admission FBC, UE, INR were normal. An elevated TSB $101.0 \mu \mathrm{mol} / \mathrm{L}$ (normal range $5-21 \mu \mathrm{mol} / \mathrm{L}$ ), Alkaline Phosphatase $504 \mathrm{U} / \mathrm{L}$ (normal range $82-383 \mathrm{U} / \mathrm{L}$ ) and GGT 189 U/L (12 - 122) was noted on LFT. A chest radiograph demonstrated a foreign body extending from L3 level, within the substance of the liver, to the T5 level in the left atrium, Figure 1A. Subsequent investigations completed after consultation with the cardiologists included an echocardiogram, computed tomographic angiogram (CTA), (Figure 1B) and contrast meal and follow through. The studies demonstrated a mild peripheral pulmonary stenosis (gradient $22 \mathrm{mmHg}$ ), confirmed the presence of the suspected UVC and an interrupted inferior vena cava (IVC). Both heterotaxy syndrome and malrotation were excluded. 
The paediatric cardiologist proceeded with endoluminal UVC retrieval, in view of the small size and young age. Vascular access was secured with a right internal jugular venous cut down. Despite unsuccessful retrieval, the UVC location was confirmed to extend from the ductus venosus to the pulmonary vein across the foramen ovale and no thrombus or vegetation was identified.

A week later after discontinuation of low molecular weight heparin and further anaesthetic assessment, bilateral inguinal

herniotomies were successfully completed as the infant remained well. Despite uneventful recovery, it was felt that the possibility of catheter removal under cardiopulmonary bypass (CPB) was not feasible due the small size of the infant.

Fourteen days later, the patient was readmitted for UVC removal in the cardiac operation theatre with the perfusionist on standby in the event CPB was indicated.

A midline upper abdominal incision was completed to accommodate for a median sternotomy if required. The umbilical vein was retrieved just distal to the portal sinus, opened between 5-0 PROLENE ${ }^{\circledR}$ (Polypropylene, ETHICON, USA) stays and traced to junction with left portal vein. The catheter was identified, grasped and easily retrieved. The tip was noted to be intact (Figure 1C). Venotomy closure with 5-0 PROLENE® (Polypropylene, ETHICON, USA) was followed by abdominal wall closure.

Postoperative recovery was complicated by a single apnoiec episode that required resuscitation. Prior to discharge, the infant was tolerating feeds and had a normal echocardiogram. At the one-month postoperative review, the infant was thriving, weighed $4.4 \mathrm{~kg}$, with a healed abdominal wound and no inguinal hernia recurrence. The patient was discharged with no further follow was required.

\section{Discussion}

Umbilical vein cannulation was first described in 1947, to facilitate an exchange transfusion in a critically ill neonate. ${ }^{1}$ Central access via the umbilical vein allows for administration of intravenous support and parenteral nutrition for a neonate in the intensive care unit. ${ }^{2}$ Reported complications associated with the insertion of umbilical vein catheters vary from infection, portal vein thrombosis, parenteral nutrition extravasation, catheter embolization, hepatic haematoma, hepatic abscess and can be fatal. ${ }^{3}$ When these UVCs fracture, timeous identification and management is focused to prevent major morbidity or even mortality.

Preventing complications starts with identifying the correct indications for UVC insertion. In addition, UVC insertion should be within 48 hours of birth when the stump is supple, easily accessible and not colonised by microbes. ${ }^{4}$ Correct anatomical positioning is also key to minimising morbidity. Ideally, after following a course from the umbilical vein into the ductus venosus to its junction with a short portion of the hepatic vein, the UVC tip should be placed at the inferior cavo-atrial junction. ${ }^{5}$

The two commonly quoted formulas that help determine the UVC length to be inserted are the Shukla formula (Length

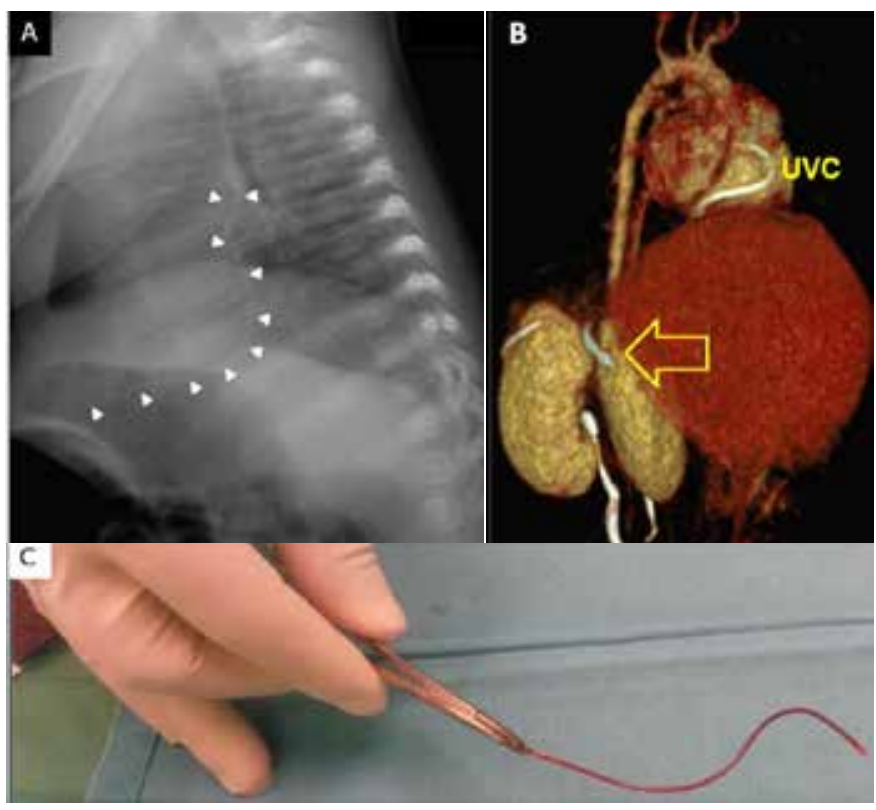

Figure 1A Pre-operative (Inguinal Herniotomy) CXR. White arrows indicating $U V C$.

L- Left; CXR - Chest radiograph; UVC- Umbilical Vein Catheter

Figure $1 B .3 D$ CTA reconstruction demonstrating the migrated UVC

3D- Three-dimensional; CTA - Computed-tomographic angiogram; UVC-umbilical vein catheter

\section{Figure 1C. Intra-op. Retrieved UVC}

$U V C$ - umbilical vein catheter

$(\mathrm{cm})=((3 \times$ Birth weight $(\mathrm{kg})+9)) / 2+1 \mathrm{~cm})^{6}$ and the Dunn method based on measurement of the shoulder-umbilicus length. ${ }^{7}$ Both formulas were described based on small cohorts and subsequent studies have demonstrated that both methods are associated with over-estimation of catheter length. $\stackrel{8}{ }$ To date there is no consensus as to which formula is most accurate for calculating the UVC length to be inserted.

On the antero-posterior view of a thoraco-abdominal radiograph, in a patient with normal anatomy, the tip position should lie at the level between the eighth and ninth thoracic vertebrae just above the right hemi-diaphragm. In two of the three cases, the UVC was identified on radiograph prior to removal. In case two, the catheter used was a feeding tube that was not radio-opaque under fluoroscopy. Modalities described to confirm UVC tip position are radiography, ultrasonography, and electrocardiography. ${ }^{9}$ Recently, UVC tip position confirmation by echocardiography was found to be superior to radiography. ${ }^{9}$

Although easy to complete, radiographs are associated with radiation exposure. Echocardiography has been shown to be more accurate and dynamic for UVC tip position localisation, determining UVC tip malposition and have no radiation exposure risk (sensitivity $56 \%$, specificity $71 \%$ ) when compared to radiography. ${ }^{9}$ However, echocardiography is not always readily available, and these results are operator 


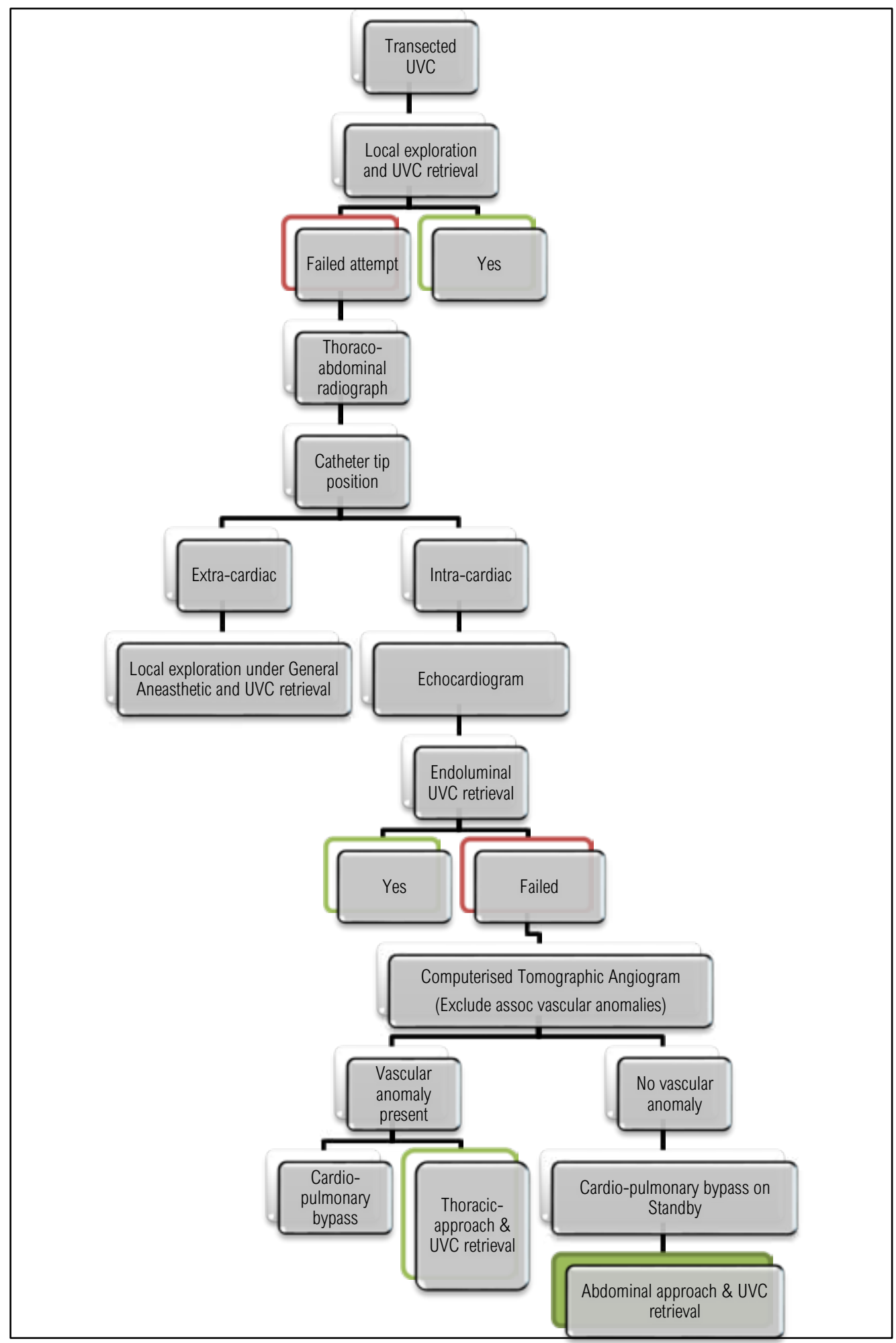

Figure 2. Management algorithm. UVC-Umbilical Vein Catheter

dependant. In case three, CTA was included as part of the diagnostic armamentarium on finding that the patient had an interrupted IVC. In addition, with the possibility of abdominal approach for UVC retrieval, a contrast meal was completed to exclude malrotation that would have been potentially corrected in the same sitting.

Ja Choi et al. ${ }^{10}$ described two mechanisms that result in UVC transection. Firstly, inadvertent damage by instruments used during catheterisation or fixation result in UVC fracture on subsequent removal. Secondly, excessive tightening of 
the purse string suture used to secure the UVC weakens the catheter wall, thus resulting in UVC fracture. In the third case, how the catheter dislodged and migrated to an intra-cardiac location is unknown. The use of fine instruments at both UVC insertion and removal can potentially prevent line fracture when used in conjunction with safe surgical technique.

In a haemodynamically stable neonate, whose FBC and INR are within normal limits, it is acceptable to proceed with exploration of the umbilical stump under local anaesthesia to retrieve the transected UVC. In most instances, this should result in successful retrieval and avoid the potential morbidity of a general anaesthesia. Unsuccessful local exploration and retrieval should prompt further clinical imaging to determine UVC position and retained length. An extra-cardiac UVC position can be retrieved via mini-laparotomy under general anaesthesia cover by the technique described. An echocardiogram is the best to identify the exact intra-cardiac UVC tip location and simultaneously evaluate for other congenital intra-cardiac anomalies. Provided there are no lifethreatening congenital cardiac lesions, endo-luminal retrieval should be attempted to avoid the morbidity of open surgery. When endoluminal retrieval fails, consideration should be given for UVC retrieval under cardio-pulmonary bypass support via thoracic or off-bypass abdominal approach as in the case of the third patient, see Figure 2.

\section{Conclusion}

The management of the transected UVC should be adapted to the patient as well as account for available resources to effect safe and successful retrieval. This process starts with considering the merits of UVC insertion, mandatory confirmation of the UVC tip position post-insertion and making a note of the UVC tip on removal. Be mindful of the potential for associated complications of UVC placement such as UVC fracture, at all points during this process and institute timeous management accordingly.

\section{Conflict of interest}

None

\section{REFERENCES}

1. Diamond LK (ed). Erythroblastosis Fetalis or Hamolytic Disease of the Newborn. Royal Society of Medicine; Royal Victoria Infirmary, Newcastle-Upon-Tyne; 1947.

2. Pasala S, Storm EA, Stroud MH, Gupta P, El-Hassan N, Lee TJ, et al. Pediatric Vascular Access and Centeses $\left(5^{\text {th }}\right.$ ed). p. 15880.e3.

3. Sherwani P, Vire A, Anand R, Jajoo M. Umbilical venous catheterization gone wrong: Hepatic complications. Indian J Radiol Imaging. 2016;26(1):40-3.

4. Mahieu LM, De Muynck AO, Ieven MM, De Dooy JJ, Goossens HJ, Van Reempts PJ. Risk factors for central vascular catheterassociated bloodstream infections among patients in a neonatal intensive care unit. J Hosp Infect. 2001;48(2):108-16.

5. Oesterich A. Umbilical vein catheterization-appropriate and inappropriate placement. Pediatr Radiol. 2010;40(12):1941-9.

6. Shukla H, Ferrara A. Rapid Estimation of Insertional Length of Umbilical Catheters in Newborns. Am J Dis Child. 1986;140(8):786-8.

7. Dunn P. Localization of the Umbilical Catheter by Post-mortem Measurement. Arch Dis Childh, 1966, 41, 69. 1966;41:69-75.

8. Mutlu M, Pariltan BK, Aslan Y, Eyupoglu I, Kader S, Akturk FA. Comparison of methods and formulas used in umbilical venous catheter placement. Turk Pediatri Ars. 2017;52(1):3542.

9. Guimaraes AF, Souza AA, Bouzada MC, Meira ZM. Accuracy of chest radiography for positioning of the umbilical venous catheter. J Pediatr (Rio J). 2017;93(2):172-8.

10. Ja Choi S, Raziuddin K, Haller JO. Broken Umbilical Artery Catheter: A Report of Two Cases. Am J Dis Child. 1977;131(5):595. 